\title{
Serum Serotonin Levels are Associated with Antiviral Therapy Outcomes in Patients with Chronic Hepatitis C
}

\author{
Jennifer M. Loftis ${ }^{*}, 1,2$, Benjamin J. Morasco ${ }^{1}$, Daniel Menasco ${ }^{2}$, Dietmar Fuchs ${ }^{3}$, Max Strater $^{2}$ and \\ Peter Hauser ${ }^{4}$
}

\author{
${ }^{I}$ Department of Psychiatry, Oregon Health \& Science University; Research \& Development Service, 3710 SW US \\ Veterans Hospital Road, Portland Veterans Affairs Medical Center, Portland, Oregon, USA \\ ${ }^{2}$ Research \& Development Service, Portland Veterans Affairs Medical Center, Portland, Oregon, USA \\ ${ }^{3}$ Department of Biological Chemistry, Biocenter, Innsbruck Medical University, Fritz Pregl Strasse 3, A-6020 \\ Innsbruck, Austria \\ ${ }^{4}$ Long Beach Veterans Affairs Medical Center, Long Beach, California, USA
}

\begin{abstract}
The role of serotonin in contributing to viral clearance has not been investigated in patients with chronic hepatitis $\mathrm{C}(\mathrm{HCV})$. In this prospective study we collected blood samples from 39 patients prior to and during antiviral therapy. Participants completed mood rating scales to monitor psychiatric symptoms, and serum serotonin and tryptophan levels were measured. Significant differences in serotonin levels were found between patients who achieved sustained viral responses (SVRs) and those who did not. Regression analysis revealed that serotonin was the only variable with a statistically significant relationship with antiviral therapy outcomes, even after controlling for other variables known to be associated with outcomes. Baseline serum serotonin levels, in combination with other variables such as degree of liver fibrosis, may be clinically useful for identifying patients in whom HCV can be cleared by antiviral therapy. Additional clinical predictors that could forecast treatment success are needed so that interventions to improve SVR rates, and reduce side effects, can be developed.
\end{abstract}

Keywords: Depression, HCV, indolamine 2, 3- dioxygenase, interferon sustained viral response, tryptophan.

\section{INTRODUCTION}

The World Health Organization estimates that about 3\% of the world population has chronic hepatitis $\mathrm{C}$ viral infection (HCV), which leads frequently to fibrosis, cirrhosis, and hepatocellular carcinoma [1]. Although HCV is treatable in some patients, there is no vaccine and the current treatment [i.e., combination therapy with pegylated interferon-alpha (IFN- $\alpha$ ) and ribavirin] has only limited efficacy. Sustained viral response (SVR) is typically achieved in only about $40-50 \%$ of those with HCV genotype 1 and $75-80 \%$ of those with HCV genotypes $2 / 3$ [2]. Thus, considerable effort has been devoted to identifying predictors of viral clearance for patients with $\mathrm{HCV}$.

Both viral (e.g., HCV genotype) and host (e.g., age [3], gender [4], body weight [5], and ethnicity [6, 7]) factors have been investigated for their roles as predictors of treatment efficacy. Among these factors, it is generally accepted that the $\mathrm{HCV}$ genotype is one variable that significantly influences $\mathrm{HCV}$ course and treatment prognosis. Specifically, infection with HCV genotype 1 is associated with more severe liver disease and a poorer response to antiviral therapy than is infection with other HCV genotypes $[8,9]$. Recently a genetic polymorphism

*Address correspondence to this author at the Portland VA Medical Center, 3710 SW US Veterans Hospital Rd., R\&D 16, Portland, OR 97239, USA; Tel: 503-220-8262, Ext. 57155; Fax: 503-273-5351;

E-mail: loftisj@ohsu.edu near the interleukin (IL) 28B gene was identified and may account for some of these differences in response to treatment [10]. However, the accuracy of predicting antiviral response based on these host factors has generally been poor [11].

In addition to limited efficacy, $\mathrm{HCV}$ treatment is also associated with significant neuropsychiatric side effects [12]. The symptoms of IFN- $\alpha$-induced depression are similar to those seen in major depressive disorder, and may include: depressed mood, irritability, emotional lability, agitation, fatigue, apathy, anhedonia, anorexia, psychomotor retardation, sleep disturbance, sexual dysfunction, memory impairment, and diminished ability to concentrate. Rates of IFN- $\alpha$-induced depression range from $0 \%$ to $44 \%[13,14]$. Although changes in the formulation of IFN- $\alpha$ have improved response efficacies for some patients, side effects of depression persist and occur in at least $20 \%$ of patients with $\mathrm{HCV}$ who receive peglylated IFN- $\alpha$ [15]. Early symptom detection and intervention can frequently prevent an adverse outcome. The depressive symptoms associated with IFN- $\alpha$ therapy are generally transient and remit following termination of therapy; however, a handful of cases have reported a worsening of depression following withdrawal from IFN- $\alpha$ therapy. In a case series of five patients treated with IFN- $\alpha$ for chronic HCV, suicide was attempted in four cases after IFN- $\alpha$ withdrawal and was responsible for two deaths [16]. In another report, two attempted suicides and one successful suicide during or 
shortly after IFN- $\alpha$ therapy for chronic HCV were described [17] (Janssen et al., 1994). Thus, given the low response rates and high rates of neuropsychiatric side effects [12], identification of the factors associated with a favorable response to treatment is a high priority.

The role of serotonin in contributing to viral clearance has not been investigated in patients with chronic HCV infection. Peripherally, serotonin mediates vascular contraction and relaxation, gastrointestinal motility, cell proliferation, apoptosis and platelet aggregation--functions that play critical roles in maintaining liver health $[18,19]$. Serotonin is present in high concentrations in platelets, and in their dense bodies, platelets store and release serotonin. Serotonin additionally acts as a growth factor $[20,21]$. In vitro and in vivo studies show that serotonin has regenerative properties in both mouse and rat livers [22-25]. However, little attention has been paid to serotonin's mechanisms of action in the human liver. Peripheral levels of serotonin and changes in tryptophan have been studied and were hypothesized to play a role in the etiology of IFN- $\alpha$-induced depression [26, 27], but serotonin has not been investigated in regards to viral clearance or liver function in patients with chronic HCV infection. Recent reports including our own findings do not support consistent correlations between IFN$\alpha$-induced depression and serotonin or tryptophan levels [2831]. Thus, given serotonin's role in immunoregulation the goals of this study were: 1) to determine the relationship between serotonin and viral clearance in patients with chronic HCV undergoing antiviral therapy, and 2) to generate hypotheses for future testing regarding the role of serotonin on liver health and regeneration.

\section{MATERIALS AND METHODOLOGY}

\section{Research Participants}

Participants were recruited through the hepatology clinics of two Veterans Affairs (VA) Medical Centers, the Portland VA Medical Center and the Seattle VA Medical Center of the Puget-Sound Health Care System between January 2003 and February 2006, for a study examining the efficacy of antidepressant therapy to prevent the development of IFN- $\alpha$ induced depression. Participants were randomly assigned to receive citalopram or placebo two weeks prior to the initiation of antiviral therapy. Participants were included in this study if they were 18 years or older, had a diagnosis of $\mathrm{HCV}$, were eligible for antiviral therapy, and agreed to undergo combination therapy with pegylated IFN- $\alpha$ and ribavirin. Participants were excluded from this study if they had ongoing depression or active psychotic symptoms during the three months prior to study start, substance abuse in the prior six months, and current use of antidepressant medication, or medical co-morbidities that could interfere with treatment. Total participant enrollment was 39 (Table 1). All of the participants completed informed consent prior to their participation in this research study. The protocol conformed to the ethical guidelines of the 1975 Declaration of Helsinki $\left(6^{\text {th }}\right.$ revision, 2008) and was approved by the Institutional Review Boards at both institutions. Additional details regarding the methods and outcomes from this randomized, double-blind, placebo-controlled trial can found in Morasco et al. [32].

\section{Procedures}

Participants were administered study measures two weeks prior to the initiation of antiviral therapy and weekly throughout treatment. At the baseline assessment, a trained research assistant administered the Structured Clinical Interview for DSM-IV (SCID), in order to assess for current psychiatric disorders. Participants also completed the Beck Depression Inventory, version II (BDI-II), a 21-item selfreport inventory of depression severity shown to have a high degree of reliability and validity [33]. Blood samples were collected at baseline (two weeks prior to the start of antiviral therapy and on the day of citalopram or placebo treatment: Week-2) and at the first day of antiviral therapy (which was two weeks after citalopram or placebo disbursement: Week 0 ). Once participants started antiviral therapy, blood was drawn every two weeks for the first three months and every four weeks for the second three months. Research study visits were coordinated with liver clinic appointments and blood samples were generally collected within a 3-hour timeframe $(11 \mathrm{am}-2 \mathrm{pm})$. Blood was drawn by venipuncture into serum separation tubes (SST) for serum determinations of serotonin and tryptophan (BD Vacutainer Systems, Franklin Lakes, NJ). The blood was centrifuged at $1500 \mathrm{RCF}$ for $20 \mathrm{~min}$ at room temperature $\left(22-25^{\circ} \mathrm{C}\right)$. Serum was collected and immediately aliquoted in polypropylene tubes (Phenix Research Products, Hayward, CA) and frozen at -80 ${ }^{\circ} \mathrm{C}$ until assayed.

\section{Antiviral Therapy}

Antiviral therapy included weekly self-administered injections of pegylated interferon- $\alpha$ (IFN- $\alpha$ ) along with twice daily oral doses of ribavirin. The doses of IFN- $\alpha$ and ribavirin were determined according to standard antiviral therapy guidelines and were monitored by a hepatology clinic provider. The intended duration of antiviral therapy was a minimum of 24 weeks (genotypes 2 and 3), although some patients continued with treatment for 48 weeks (genotype 1). Patients were given the option to discontinue treatment at any time.

\section{Administration and Monitoring of Citalopram or Placebo}

A double-blind, randomized block design was used to assign participants to an experimental condition: citalopram [selective serotonin reuptake inhibitor antidepressant (SSRI)] or placebo. Stratification was based on the geographical location of the study. Upon study entry, $20 \mathrm{mg}$ tablets of citalopram or identical appearing tablets of the placebo were blindly dispensed to participants in the respective study arms. Participants were asked to self-administer one of the oral tablets on a daily basis. Depressive symptoms were monitored using rating scales at each clinical visit and the severity of depression, as measured by BDI-II scores, determined whether dose increases were necessary. After 24 weeks, the code for treatment group assignment was broken for all participants and those who continued with antiviral therapy for 48 weeks were offered the option of open-label citalopram for the duration of antiviral therapy. Four patients in the placebo group and three patients in the SSRI group developed moderate depression and entered the rescue arm of the study. For these seven patients, the code for treatment 
Table 1. Comparison of Demographic Characteristics

\begin{tabular}{|c|c|c|c|c|}
\hline Age, M (SD) & $53.0(7.3)$ & $50.7(7.5)$ & $54.8(6.8)$ & 0.08 \\
\hline Male & $36(92.3 \%)$ & $16(94.1 \%)$ & $20(90.9 \%)$ & \\
\hline Female & $3(7.7 \%)$ & $1(5.9 \%)$ & $2(9.1 \%)$ & \\
\hline Caucasian & $33(84.6 \%)$ & $15(88.2 \%)$ & $18(81.8 \%)$ & \\
\hline Other & $6(15.4 \%)$ & $2(11.8 \%)$ & $4(18.2 \%)$ & \\
\hline Education & & & & 0.04 \\
\hline$\leq$ High School Diploma & $13(33.3 \%)$ & $9(52.9 \%)$ & $4(18.2 \%)$ & \\
\hline Retired & $8(20.5 \%)$ & $4(23.5 \%)$ & $4(18.2 \%)$ & \\
\hline Other & $11(28.2 \%)$ & $7(41.2 \%)$ & $4(18.2 \%)$ & \\
\hline Genotype & & & & 0.53 \\
\hline 1 & $21(53.8 \%)$ & $8(47.1 \%)$ & $13(59.1 \%)$ & \\
\hline 2 or 3 & $18(46.2 \%)$ & $9(52.9 \%)$ & $9(40.9 \%)$ & \\
\hline Baseline HCV Load & & & & 0.55 \\
\hline Low & $15(40.5 \%)$ & $5(13.5 \%)$ & $10(27.0 \%)$ & \\
\hline Moderate & $11(29.7 \%)$ & $5(13.5 \%)$ & $6(16.2 \%)$ & \\
\hline Current Depression Severity, M (SD) & $4.3(4.3)$ & $3.7(3.5)$ & $4.7(4.9)$ & 0.47 \\
\hline
\end{tabular}

group assignment was broken prior to week 24 and they either started on citalopram (placebo group) or received medication adjustments (SSRI group).

\section{Liver Health Laboratory Measures}

For all research participants liver health indices [i.e., platelet counts, SVRs, and aspartate aminotransferase (AST) values] were collected from each veteran's electronic medical record. HCV viral load was determined using the Ortho VITROS chemiluminescence assay (CIA) method for detection of antibodies to $\mathrm{HCV}$. Sustained viral response (SVR) was defined as undetectable $\mathrm{HCV}$ at 6 months following the completing of antiviral therapy. APRI scores were calculated based on $\underline{A}$ ST to Platelet Ratio Index and were used as an assessment of liver fibrosis.

\section{Serotonin Assays}

High performance liquid chromatography (HPLC) was used to measure serum serotonin levels. A modified Waters Corp Oasis MCX method was used to extract serotonin from human serum. Both samples and standards selected for analysis were thawed at $4^{\circ} \mathrm{C}$ prior to Solid Phase Exchange (SPE) workup. Prior to SPE workup a $200 \mu \mathrm{L}$ aliquot of sample serum was prepared with $10 \%$ ascorbic acid $[\mathrm{w} / \mathrm{v}], 5-$ hydroxytryptophan (5-HTP) internal standard $(150 \mathrm{ng} / \mathrm{mL})$, and $1 \%$ formic acid (aq). Endogenous levels of serum serotonin (5-HT) were calculated using a linear range of 5HT standards consisting of 7.5, 15, 50, 150, 500 and 2500 $\mathrm{ng} / \mathrm{mL}$. All standards were prepared and treated identically to samples; however, standards used $200 \mu \mathrm{L}$ of 5-HT-free serum (Valley Biomedical, Winchester, VA, USA). The $30 \mathrm{mg}$ MXC cartridges (Waters, Milford, MA, USA) were equilibrated and conditioned with $1 \mathrm{~mL}$ of both methanol and $1 \%$ formic acid (aq), respectively. After sample application the cartridges were washed with $1 \mathrm{~mL}$ of $0.1 \mathrm{~N} \mathrm{HCl}$ (aq) followed by $1 \mathrm{~mL}$ of methanol. Serotonin was removed from the SPE cartridge with $1.5 \mathrm{~mL}$ of $7.5 \% \mathrm{NH}_{4} \mathrm{OH}$ in methanol. The eluent was collected and desiccated under nitrogen and reconstituted in a 7:1 solution of mobile phase and an 
aqueous antioxidant mixture containing $1.0 \mathrm{mM}$ oxalic acid, 3.0mM L-cysteine, and 0.1 M acetic acid [34].

The HPLC system consisted of an ESA Biosciences model 580 single channel pump, 540 autosampler, and an ESA Coulochem II electrochemical detector (ESA Biosciences, Milford, MA, USA). The mobile phase buffer was prepared by filtering $\mathrm{NaH}_{2} \mathrm{PO}_{4} \cdot \mathrm{H}_{2} \mathrm{O}(30 \mathrm{mM})$ and sodium citrate $(14.7 \mathrm{mM})$ with a $0.45 \mu \mathrm{m}$ nylon filter in $18 \mathrm{M} \Omega$ Milli-Q water. Both $\mathrm{Na}_{2}$ EDTA $(2.7 \mathrm{~mm})$ and 1octanesulfonic acid $(2.23 \mathrm{mM})$ were subsequently added whereupon the mobile phase $\mathrm{pH}$ was adjusted to 3.5 with ophosphoric acid; the addition of the organic modifiers $8 \%$ acetonitrile and 1\% tetrahydrofuran, immediately preceded followed by degassing (Supelco, Bellefonte, PA, USA) using a $1.5 \mu \mathrm{m}$ Teflon membrane under vacuum [35]. Injections of $20 \mu 1$ were isocratically chromatographed at $0.6 \mathrm{~mL} / \mathrm{min}$ over a $3.2 \mu \mathrm{m}, 3.0 \times 150-\mathrm{mm}$ reverse phased $\mathrm{C}_{18}$ column with 4.0x10-mm guard (ESA Biosciences, Milford, MA, USA) at $25^{\circ} \mathrm{C}$ for twenty minutes. The electrochemical 5020 ESA guard cell potential was $+350 \mathrm{mV}$ while both ESA $5011 \mathrm{~A}$ analytical electrodes E1 and E2 (ESA Biosciences, Milford, MA, USA) potentials were experimentally determined by Hydrodynamic Voltammogram (data not shown) at $50 \mathrm{mV}$, Range $(1 \mu \mathrm{A})$, and $+200 \mathrm{mV}$, Range $(100 \mathrm{nA})$, respectively. All cued samples were incubated at $4{ }^{\circ} \mathrm{C}$ and injected in duplicate by the 540 autosampler.

Chromatographic isolation of both 5-HTP and 5-HT from other potential interfering compounds was achieved as evidenced by their average retention times of 2.8 and 11.7minutes, respectively. External calibration curve intraprecision indices of linearity averaged $\mathrm{R}^{2}=0.9996 \pm 0.3 \mathrm{E}-3$ (SD) yielding a coefficient of variation $(\mathrm{CV})$ of $0.03 \%$. The population average percent recovery of 5-HTP was $90.0 \% \pm$ 6.8 (SD) and yielded a CV of 7.6\% $(n=32)$. Limits of detection were $\geq 7.5 \mathrm{ng} / \mathrm{mL}$ at operating potentials with a signal-to-noise ratio of 47.8 .

\section{Tryptophan Assay}

Tryptophan concentrations were measured by HPLC on reversed phase $\mathrm{C} 18$ material using 3-nitro-L-tyrosine as internal standard, as previously described. Briefly, serum protein precipitated with $2 \mathrm{mmol} / \mathrm{L}$ trichloroacetic acid and tryptophan was monitored by its natural fluorescence at 285 $\mathrm{nm}$ excitation and $350 \mathrm{~nm}$ emission wavelengths [36, 37].

\section{Statistical Analysis}

Baseline demographic differences between the two groups (participants who achieved SVR versus those who did not achieve SVR) were compared using chi-square tests for categorical variables and analysis of variance (ANOVA) for continuous variables. The effects of antiviral therapy on peripheral serotonin and tryptophan levels were assessed with analyses of covariance (ANCOVAs). In these analyses, education level was a covariate, as it significantly differed between the two groups at the baseline assessment. Separate analyses were conducted for serotonin and for tryptophan. ANOVAs were also conducted to evaluate whether randomization to citalopram or placebo had an impact on serotonin or tryptophan levels, at different time points. Finally, hierarchical logistic regression was used to evaluate variables associated with antiviral treatment outcome. Body mass index (BMI) and level of education were entered in Step 1. In Step 2, HCV genotype, baseline viral load counts (defined as low 0-1,569,999; moderate 1,570,000-4,129,999; or high $4,130,000$ or more copies $/ \mathrm{mL}$ ), and whether or not patients had cirrhosis (yes or no) were entered into the equation. Note: viral loads are reported in copies per $\mathrm{mL}$ as opposed to International Units per $\mathrm{mL}(\mathrm{IU} / \mathrm{mL})$. Given that these lab values were collected prior to the study onset using differing clinical laboratory assays, applying a standard conversion factor to the values was not appropriate [38]. Viral load was placed into categorical data as the original data were not normally distributed. For Step 3, baseline serotonin and tryptophan levels were entered. Genotype, viral load, cirrhosis status and education were categorical variables, while BMI and serotonin and tryptophan levels were continuous. Results were considered statistically significant if $P \leq 0.05$, two-tailed. This study had power greater than $80 \%$ to detect a 10 -point difference in scores on the BDI-II in 39 patients. Calculations were performed using SPSS software.

\section{RESULTS}

Patient demographics are reported in Table 1, including HCV genotype, BMI, viral load at baseline, and other variables putatively associated with antiviral therapy outcome. Significant differences between patients who achieved SVR and those who did not were found only for level of education (Table 1), as the SVR group had less education.

\section{Association Between Serum Serotonin Levels and Antiviral Therapy Response}

Higher peripheral serotonin levels were significantly associated with improved treatment outcomes in patients undergoing antiviral therapy before and during treatment. At week-2 $[F(2,26)=6.15, P=0.02]$, week $12[F(2,17)=$ $4.45, P=0.05]$ and week $24[F(2,20)=6.65, P=0.02]$, the patients who had undetectable HCV at six months following the completion of treatment (SVR group) had significantly higher serum serotonin levels as compared with patients who didn't achieve an SVR (no SVR group) (Fig. 1A). To determine whether the differences in serotonin and SVR rates were associated with changes in the bioavailability of serotonin's precursor, tryptophan levels were also measured. Patients who achieved viral clearance did not have significantly different levels of tryptophan than patients who did not achieve an SVR (Fig. 1B).

Patients randomly assigned to the SSRI group displayed significantly lower serotonin levels than patients randomized to the placebo group at week $0[F(1,24)=24.6, P<0.001]$, week $2[F(1,21)=30.2, P<0.001]$, week $8[F(1,14)=$ $16.5, P=0.001]$, week $12[F(1,19)=12.9, P=0.002]$, week $16[F(1,13)=10.6, P=0.007]$ and week $24[F(1,22)$ $=14.6, P=0.001]$ (Fig. 2A). Patients in the SSRI group were not statistically less likely to achieve SVRs, as compared to patients in the placebo group, but the SVR rate in the SSRI group was notably lower when compared with the placebo group $\left[\begin{array}{lllll}36.8 \% & (7 / 19) & v s & 50 \% & (10 / 20) \text {, }\end{array}\right.$ respectively][32]. No significant differences in tryptophan levels were detected during the course of antiviral therapy between patients in the SSRI and placebo groups (all $P$ values $\geq 0.05$; Fig. 2B). 

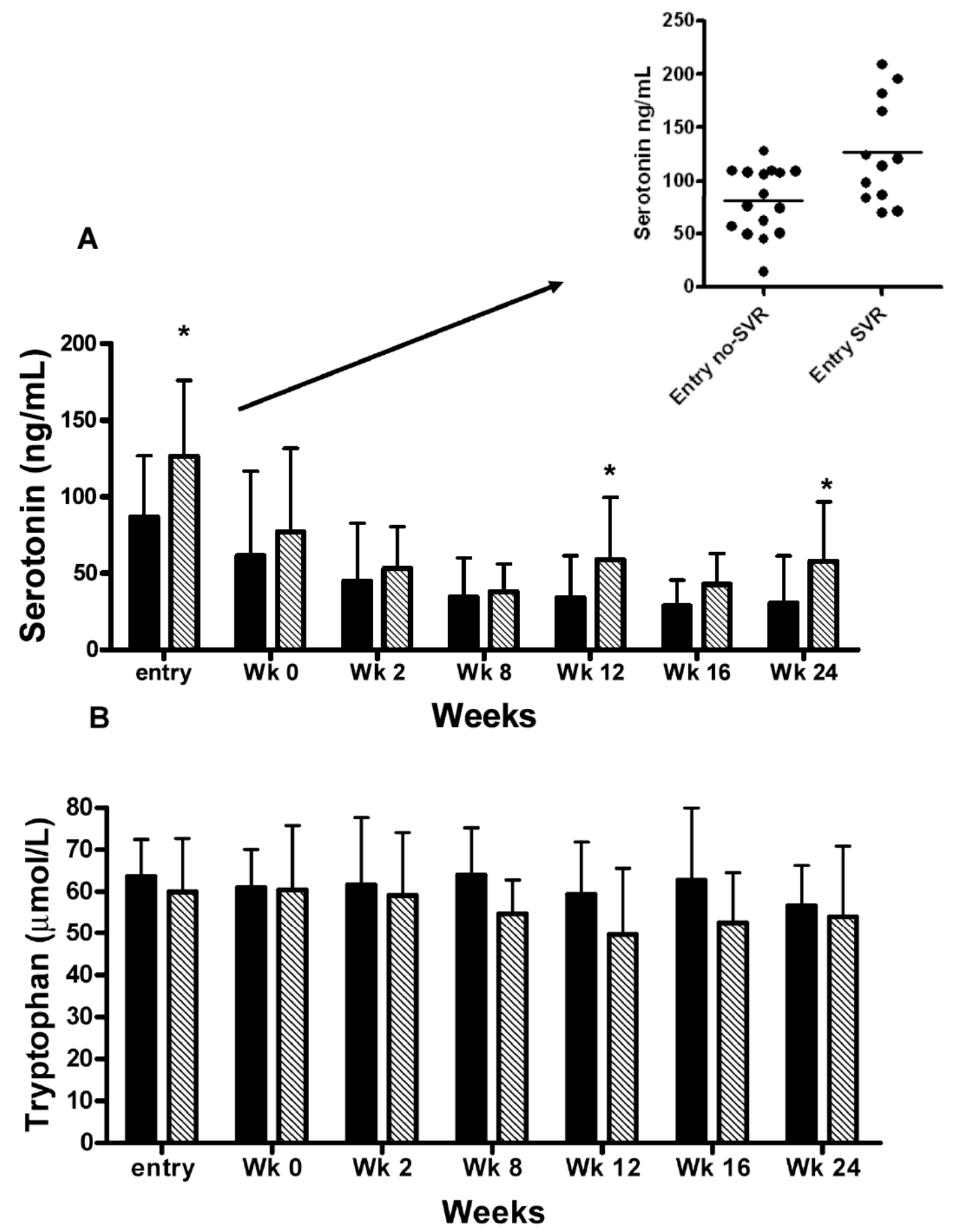

Fig. (1). Higher peripheral serotonin levels are associated with improved antiviral therapy outcomes in patients undergoing treatment for $\mathrm{HCV}$. Serum samples were analyzed for serotonin and tryptophan levels in patients who did and did not achieve sustained viral responses six months after the completion of therapy (SVR, $\mathrm{n}=17$ and no SVR, $\mathrm{n}=22)$. For serotonin levels, significant differences were found between the groups at study entry $(\mathrm{P}=0.02)$, week $12(\mathrm{P}=0.05)$ and week $24(\mathrm{P}=0.02)(\mathrm{A})$. The same relationship was not evident for tryptophan levels and antiviral therapy outcomes (B). Solid bars represent the no SVR group and cross-hatched bars represent the SVR group. ARUP Laboratories, a National Reference Laboratory (Salt Lake City, UT), report that the average reference interval for serotonin levels in serum is $50-220 \mathrm{ng} / \mathrm{mL}$. Data are expressed as means \pm standard deviations (SD).

Baseline Serotonin Level as a Predictor of Viral Response in HCV Patients

Table 2 shows results from the hierarchical logistic regression examining variables putatively associated with antiviral therapy outcome. Step 1, with education levels and
BMI included, was not significant, $\chi^{2}(d f=2, n=24)=0.70, P$ $=0.70$. For Step 2, liver health (i.e., presence or absence of cirrhosis and HCV load) and viral (i.e., HCV genotype) factors were included and were not significant, $\chi^{2}(d f=8$, $n=24)=7.08, P=0.53$. The inclusion of serotonin and tryptophan levels in Step 3 evidenced a trend toward 

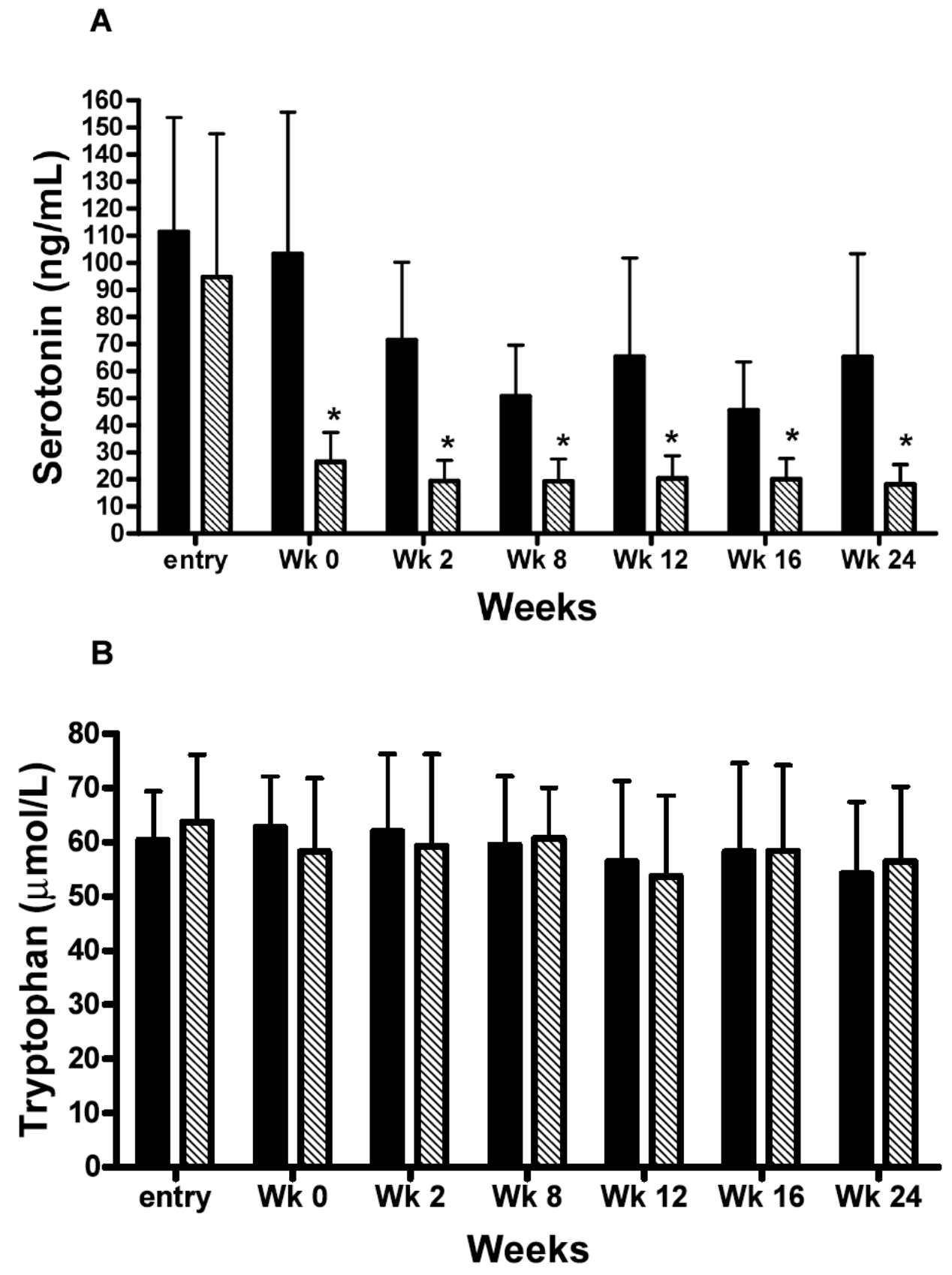

Fig. (2). Serotonin levels are significantly lower in patients on citalopram as compared to placebo. Patients with HCV were randomly assigned to SSRI (citalopram, $\mathrm{n}=14$ ) or placebo $(\mathrm{n}=15)$ two weeks prior to starting antiviral therapy. Significant differences in serotonin levels were found between SSRI and placebo groups at week $0(\mathrm{P}<0.001)$, week $2(\mathrm{P}<0.001)$, week $8(\mathrm{P}=0.001)$, week $12(\mathrm{P}=0.002)$, week $16(\mathrm{P}=0.007)$ and week $24(\mathrm{P}=0.001)(\mathrm{A})$. However, patients in the SSRI group were not less likely to achieve sustained viral response rates, as compared to patients in the placebo group. The SVR rate in the SSRI group was 36.8\% (7/19) compared to 50\% (10/20) in the placebo group $(\mathrm{P}=0.41)$. No significant differences in tryptophan levels were detected $(\mathbf{B})$. Solid bars represent the placebo group and cross-hatched bars represent the SSRI group. Data are expressed as means \pm SD.

significance, $\chi^{2}(d f=2, n=24)=5.62, P=0.06$, but the overall model was not significant, $\chi^{2}(d f=8, n=24)=9.87, P=0.27$. Baseline serotonin level was the only variable with a statistically significant relationship with antiviral therapy outcome $(P=0.05)$.

\section{Blood Platelet Numbers are Slightly Higher in Patients with SVRs}

Baseline platelet numbers were compared between patients who did and did not achieve SVR. Patients who achieved an SVR had higher numbers of platelets as compared with patients who did not; however, this difference was not statistically significant $(P=0.55$; means \pm SD: $192.89 \pm 74.55 \mathrm{~K} / \mathrm{cmm}$ vs $174.71 \pm 67.58 \mathrm{~K} / \mathrm{cmm}$, respectively). Because platelet counts are lower than normal in patients with liver cirrhosis, we tested the possibility that differences in degree of liver damage also influenced SVR. Unlike serotonin levels, the presence or absence of cirrhosis was not a statistically significant predictor of SVR (Tables 1 and 2). 
Table 2. Logistic Regression Analysis Predicting Antiviral Therapy Outcome

\begin{tabular}{|l|c|c|c|c|}
\hline \multicolumn{1}{|c|}{ Beta (SD) } & Wald & Significance & Odds Ratio 95\% (Confidence Interval) \\
\hline \hline Step 1 & $0.01(0.09)$ & 0.01 & 0.92 & $2.1(0.4-11.6)$ \\
\hline BMI & $0.72(0.88)$ & 0.67 & 0.42 & $2.6(0.2-37.0)$ \\
\hline Education & $0.96(1.35)$ & 0.50 & 0.48 & $0.6(0.1-3.3)$ \\
\hline Step 2 & $-0.60(0.92)$ & 0.43 & 0.51 & $1.1(0.3-4.2)$ \\
\hline Liver cirrhosis & $0.13(0.67)$ & 0.04 & 0.85 & $1.1(1.0-1.1)$ \\
\hline HCV genotype & & & \\
\hline Viral load & $0.03(0.01)$ & 3.90 & 0.05 & $0.9(0.8-1.1)$ \\
\hline Step 3 & $-0.05(0.06)$ & 0.59 & 0.44 & \\
\hline Serotonin &
\end{tabular}

\section{Serotonin Levels Correlate with Fibrosis Scores}

Fig. (3) illustrates a significant negative correlation between serotonin levels and APRI scores [an assessment of liver fibrosis; aspartate aminotransferase (AST) to platelet ratio index], such that higher serotonin levels at entry were associated with lower APRI scores and putatively less liver fibrosis. Serotonin levels were significantly correlated with APRI values suggesting that higher serotonin levels might be a surrogate marker for less advanced liver disease.

\section{Depressive Symptoms During Antiviral Therapy are Not Directly Correlated with Serotonin and Tryptophan Levels}

To test the hypothesis that IFN- $\alpha$-induced decreases in serotonin or tryptophan are associated with an increase in depressive symptoms, BDI-II scores were correlated with serotonin and tryptophan levels before and during antiviral therapy--at week-2, week 0 , week 2 , week 8 , week 12 , week 16 and week 24 (Table 3). Pearson correlations were conducted, and tryptophan was significantly correlated with
BDI-II scores at week $8(r=0.55, P=0.04)$, while serotonin was significantly correlated with BDI-II scores at week $12(r$ $=0.50, P=0.02)$. Statistically significant negative correlations were not found between BDI-II scores and tryptophan or serotonin levels.

\section{DISCUSSION}

During a prophylactic antidepressant study that was conducted to evaluate the safety and efficacy of pre-treating patients with citalopram, an SSRI antidepressant, prior to and during antiviral therapy [32], a novel role for serotonin was discovered. The concept that serotonin may play a role in hepatic wound healing is growing in interest and several recent empirical studies show the benefits of serotonin in rodent liver regeneration [19, 39]. The liver regenerative properties of serotonin may be of particular importance in chronic HCV, where the virus attacks the liver through the lytic cycle destroying hepatocytes, which could be regenerated via platelet-derived serotonin or specific serotonin agonists $[22,23]$. Proinflammatory cytokines may increase the availability of hepatic serotonin [40], but

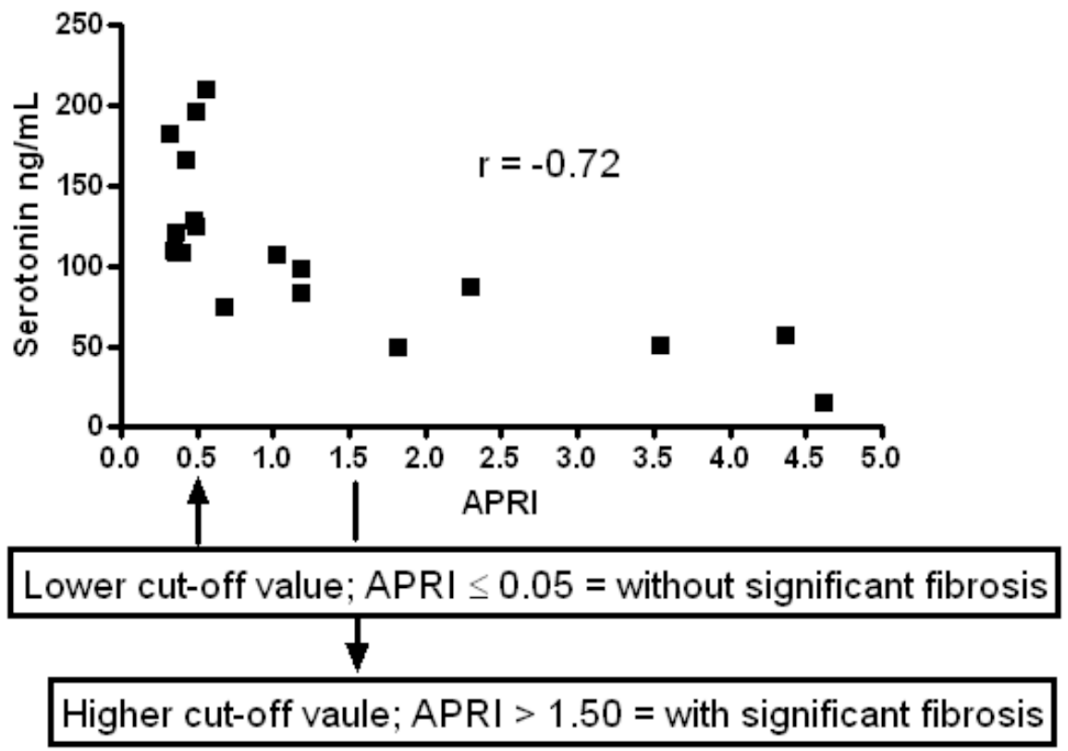

Fig. (3). Correlation between serotonin levels and APRI values (Pearson $r=-0.72, P=0.0005$ ). 
Table 3. Pearson Correlations Between BDI-II Scores and Serum Biomarkers in Patients with HCV Undergoing Antiviral Therapy

\begin{tabular}{|c|c|c|c|c|c|}
\hline Week & BDI-II Score Mean (SD) & Serotonin (ng/mL) Mean (SD) & Tryptophan $(\mu$ mol/L) Mean (SD) & Serotonin $(\boldsymbol{r})$ & Tryptophan $(\boldsymbol{r})$ \\
\hline \hline-2 & $4.28(4.35)$ & $95.15(55.51)$ & $62.04(10.69)$ & -0.06 & -0.02 \\
\hline 0 & $3.54(3.35)$ & $66.47(54.40)$ & $60.83(11.26)$ & -0.16 & 0.25 \\
\hline 2 & $6.23(5.32)$ & $47.83(34.07)$ & $60.69(15.33)$ & 0.26 & 0.12 \\
\hline 8 & $7.18(6.10)$ & $36.11(21.69)$ & $60.08(10.73)$ & $0.50^{*}$ & 0.11 \\
\hline 12 & $6.72(6.18)$ & $45.16(35.46)$ & $55.00(14.60)$ & 0.15 & -0.03 \\
\hline 16 & $7.36(6.43)$ & $34.67(19.14)$ & $58.28(15.59)$ & -0.13 & -0.41 \\
\hline 24 & $5.33(4.79)$ & $44.83(37.20)$ & $55.40(13.24)$ & & \\
\hline
\end{tabular}

serotonin may also contribute to improved viral clearance by enhancing the release of cytokines at the site of liver injury $[41,42]$. The molecular mechanisms regarding how serotonin aids in HCV clearance are yet to be determined.

Not all research supports the theory that serotonin aids in hepatic wound healing, as serotonin has been shown in some animal studies to contribute to liver injury following intestinal and hepatic ischemia-reperfusion [43, 44]. Further, Lang et al. recently reported that serotonin contributes to viral persistence in mice infected with the noncytopathic lymphocytic choriomeningitis (LCMV) [45]. More specifically, $\mathrm{CD}^{+} \mathrm{T}$ cell antiviral response was delayed by serotonin treatment and replication of LCMV was enhanced in serotonin treated mice [45]. In contrast to the effects of serotonin in their mouse model of LCMV-induced hepatitis, we found that higher serotonin levels were significantly associated with improved antiviral therapy outcomes in patients with HCV undergoing antiviral therapy (Fig. 1A). Our findings are in agreement with a recent study by Manéglier et al. who used human cells to show that serotonin may also help in human immunodeficiency viral (HIV) infections [46]. The authors found that serotonin decreases HIV-1 infection most likely via a decrease in the expression of CCR5 [HIV-1/Ba-L receptor (CD4) coreceptor] and/or an increase in the expression of CCR5's ligand, macrophage inflammatory protein-1alpha (MIP-1 $\alpha$ ) [46]. Although serotonin's mechanisms of action in aiding viral clearance are likely different for $\mathrm{HCV}$ and HIV infections, collectively these findings support a vital role for platelet serotonin in contributing to these processes in humans [18].

Peripheral levels of serotonin and changes in tryptophan have been studied and were hypothesized to play a role in the etiology of the neuropsychiatric side effects associated with antiviral therapy, particularly depression [27, 28], but more recent reports including our own findings do not support consistent correlations between IFN- $\alpha$-induced depression and serotonin or tryptophan levels [28-31, 47]. In agreement with Fontana et al., we also found significant IFN- $\alpha$-induced decreases in peripheral serotonin levels (most notably in the SSRI group), which did not negatively correlate with depression rating scale scores (Table 3) [31]. Given that both proinflammatory cytokines (e.g., interferons) and $\mathrm{HCV}$ are associated with an upregulation of indolamine 2,3 dioxygenase (an enzyme which converts tryptophan into kynurenine) [48], further research is needed to determine more sensitive markers of serotonin metabolism such as serotonin gene polymorphisms $[49,50]$ which could be used to predict or influence IFN- $\alpha$-induced depression.

Robaeys et al. recently asked an important question about whether or not the use of antidepressant medications reduces the efficacy of interferon (IFN)-based therapies in patients with hepatitis $\mathrm{C}(\mathrm{HCV})$ undergoing antiviral treatment [51]. Kraus et al. aptly responded by emphasizing the role for serotonin: 1) in the hypothesized etiology of IFN-induced depression and 2) in the use of selective serotonin reuptake inhibitor (SSRI) antidepressants to treat IFN-induced depression [52]. Serotonin's role in liver health and regeneration is an additional consideration. We report that patients with HCV who achieved sustained viral responses (SVRs) six months following the completion of therapy had significantly higher serotonin levels prior to and during IFN therapy, as compared to patients who didn't achieve SVRs (Table 2; Fig. 1A). Further, patients who were prophylactically prescribed SSRIs prior to IFN therapy displayed significantly lower serotonin levels than patients in the placebo group (Fig. 2A). Although patients in the SSRI group were not statistically less likely to achieve SVRs, as compared to patients in the placebo group, the SVR rate in the SSRI group was notably lower when compared with the placebo group [7/19 (36.8\%) and 10/20 (50\%), respectively]. Serotonin levels were also significantly correlated with the estimated degree of liver fibrosis (Fig. 3), suggesting that higher serotonin levels might be a surrogate marker for less advanced liver disease, and consequently improved $\mathrm{HCV}$ treatment outcomes. Our findings do not provide an answer to the questions raised by Robaeys et al. and Kraus et al. [51, 52], but the data do support the need for more research regarding the effects of serotonin on liver health and regeneration in humans.

There exist several limitations to the current results. In general, the majority of patients in this research study experienced only modest increases in depressive symptoms during the course of antiviral therapy. A greater degree of psychopathology may be needed in order to identify and test causal relationships between IFN- $\alpha$-induced changes in serotonin and tryptophan levels and depression. A concern also exists regarding power, as the sample size was low to detect significant effects. In addition, our study used the APRI as a measure of disease severity, which has been 
validated in $\mathrm{HCV}$ patients $[53,54]$ but has limited sensitivity and specificity for values of 0.5 [55]. The APRI is a less reliable estimate of liver disease severity than liver biopsies or measures of portal vein hypertension, which were beyond the resources of this study to obtain. Finally, we recruited our sample of patients from two VA hospitals, and it was composed almost exclusively of men. Thus, the results may not generalize to other clinical populations. Despite these concerns, there are also several strengths of the present study, including the prospective design, randomization scheme, and collection of blood and psychological measures at multiple time points.

\section{CONCLUSIONS}

In conclusion, these preliminary findings suggest that higher peripheral serotonin levels are associated with improved antiviral treatment outcomes in patients with $\mathrm{HCV}$. These results should be replicated, as they are based on secondary analysis from the original randomized clinical trial, but may have clinical implications regarding the use of SSRIs in patients with $\mathrm{HCV}$ and other liver diseases.

\section{ACKNOWLEDGEMENTS}

This research study was supported by the National Institute for Mental Health grant F32 MH071137-01 (to J.M.L.), the Veteran's Health Administration Merit Review grant (to P.H.), National Institute on Drug Abuse (award K23DA023467 to B.J.M.), and by the Stiftung Propter Homines, Vaduz-Fürstentum Liechtenstein" (to D.F.). The authors also wish to acknowledge the support of the Department of Veteran Affairs Northwest Hepatitis C Resource Center Program. The content of this manuscript is the responsibility of the authors and does not necessarily represent the official views of the Department of Veterans Affairs or the National Institutes of Health.

\section{CONFLICT OF INTEREST}

The authors have no conflicts of interest to report.

\section{REFERENCES}

[1] Hepatitis C: Global prevalence. World Health Organization. Wkly Epidemiol Rec 1997; 72: 341-48.

[2] Hauser P, Morasco BJ, Linke A, et al. Antiviral completion rates and sustained viral response in hepatitis $C$ patients with and without preexisting major depressive disorder. Psychosomatics 2009; 50: 500-5.

[3] Forns X, Bukh J. The molecular biology of hepatitis $\mathrm{C}$ virus. Genotypes and quasispecies. Clin Liver Dis 1999; 3(vii): 693-716.

[4] Hu KQ, Vierling JM, Redeker AG. Viral, host and interferonrelated factors modulating the effect of interferon therapy for hepatitis C virus infection. J Viral Hepat 2001; 8: 1-18.

[5] Hourigan LF, Macdonald GA, Purdie D, et al. Fibrosis in chronic hepatitis C correlates significantly with body mass index and steatosis. Hepatology 1999; 29: 1215-9.

[6] Howell C, Jeffers L, Hoofnagle JH. Hepatitis C in African Americans: summary of a workshop. Gastroenterology 2000; 119: 1385-96.

[7] Reddy KR, Hoofnagle JH, Tong MJ, et al. Racial differences in responses to therapy with interferon in chronic hepatitis C. Consensus Interferon Study Group. Hepatology 1999; 30: 787-93.

[8] Le Guen B, Squadrito G, Nalpas B, Berthelot P, Pol S, Brechot C. Hepatitis $C$ virus genome complexity correlates with response to interferon therapy: a study in French patients with chronic hepatitis C. Hepatology 1997; 25: 1250-4.

[9] Gervais A, Martinot M, Boyer N, et al. Quantitation of hepatic hepatitis C virus RNA in patients with chronic hepatitis C.
Relationship with severity of disease, viral genotype and response to treatment. J Hepatol 2001; 35: 399-405.

[10] Ge D, Fellay J, Thompson AJ, et al. Genetic variation in IL28B predicts hepatitis $C$ treatment-induced viral clearance. Nature 2009; 461(7262): 399-401.

[11] Davis GL, Lau JY. Factors predictive of a beneficial response to therapy of hepatitis C. Hepatology 1997; 26: 122S-7S.

[12] Loftis JM, Hauser P. The phenomenology and treatment of interferon-induced depression. J Affect Disord 2004; 82: 175-90.

[13] Matsushita E, Unoura M, Kobayashi K. Psychiatric complications of interferon therapy. Nippon Rinsho 1994; 52, 1910-3.

[14] Miyaoka H, Otsubo T, Kamijima K, Ishii M, Onuki M, Mitamura $\mathrm{K}$. Depression from interferon therapy in patients with hepatitis C. Am J Psychiatry 1999; 156: 1120.

[15] Fried MW, Shiffman ML, Reddy KR, et al. Peginterferon alfa-2a plus ribavirin for chronic hepatitis $\mathrm{C}$ virus infection. $\mathrm{N}$ Engl $\mathrm{J}$ Med 2002; 347: 975- 82.

[16] Rifflet H, Vuillemin E, Oberti F, et al. Suicidal impulses in patients with chronic viral hepatitis $\mathrm{C}$ during or after therapy with interferon alpha. Gastroenterol Clin Biol 1998; 22: 353- 7.

[17] Janssen H, Brouwer J, van der Mast R, et al. Suicide associated with alfa-interferon therapy for chronic viral hepatitis. J. Hepatol 1994; 21, 241- 3 .

[18] Ruddell RG, Mann DA, Ramm GA. The function of serotonin within the liver. J Hepatol 2008; 48: 666-75.

[19] Lesurtel M, Soll C, Graf R, Clavien PA. Role of serotonin in the hepato-gastroIntestinal tract: an old molecule for new perspectives. Cell Mol Life Sci 2008; 65: 940-52.

[20] Seuwen K, Pouyssegur J. Serotonin as a growth factor. Biochem Pharmacol 1990; 39: 985-90.

[21] Fanburg BL, Lee SL. A new role for an old molecule: serotonin as a mitogen. Am J Physiol 1997; 272: L795-L806.

[22] Balasubramanian S, Paulose CS. Induction of DNA synthesis in primary cultures of rat hepatocytes by serotonin: possible involvement of serotonin S2 receptor. Hepatology 1998; 27: 62-6.

[23] Lesurtel M, Graf R, Aleil B, et al. Platelet-derived serotonin mediates liver regeneration. Science 2006; 312: 104-7.

[24] Papadimas GK, Tzirogiannis KN, Panoutsopoulos GI, et al. Effect of serotonin receptor 2 blockage on liver regeneration after partial hepatectomy in the rat liver. Liver Int 2006; 26: 352-61.

[25] Tzirogiannis KN, Panoutsopoulos GI, Papadimas GK, et al. The effect of hepatic stimulator substance (HSS) on liver regeneration arrest induced by 5-HT2 receptor blockade. In Vivo 2005; 19: 8417.

[26] Bonaccorso S, Marino V, Puzella A, et al. Increased depressive ratings in patients with hepatitis $\mathrm{C}$ receiving interferon-alpha-based immunotherapy are related to interferon-alpha-induced changes in the serotonergic system. J Clin Psychopharmacol 2002; 22: 86-90.

[27] Capuron L, Ravaud A, Neveu PJ, et al. Association between decreased serum tryptophan concentrations and depressive symptoms in cancer patients undergoing cytokine therapy. Mol Psychiatry 2002; 7: 468-73.

[28] Wichers MC, Koek GH, Robaeys G, et al. IDO and interferonalpha-induced depressive symptoms: a shift in hypothesis from tryptophan depletion to neurotoxicity. Mol Psychiatry 2005; 10: 538-44.

[29] Russo S, Kema IP, Haagsma EB, et al. Irritability rather than depression during interferon treatment is linked to increased tryptophan catabolism. Psychosom Med 2005; 67: 773-7.

[30] Van Gool AR, Verkerk R, Fekkes D, et al. Neurotoxic and neuroprotective metabolites of kynurenine in patients with renal cell carcinoma treated with interferon-alpha: course and relationship with psychiatric status. Psychiatry Clin Neurosci 2008; 62: 597-602.

[31] Fontana RJ, Kronfol Z, Lindsay KL, et al. Changes in mood states and biomarkers during peginterferon and ribavirin treatment of chronic hepatitis C. Am J Gastroenterol 2008; 103: 2766-75.

[32] Morasco BJ, Loftis JM, Indest DW, et al. Prophylactic antidepressant treatment in patients with hepatitis $\mathrm{C}$ on antiviral therapy: A double-blind, placebo-controlled trial. Psychosomatics 2010; 51(5): 401-8.

[33] Beck A, Steer R, Brown G. Beck Depression Inventory. In: Brace $H$, Eds. Beck Depression Inventory, $2^{\text {nd }}$ ed Manual. Texas: The Psychological Corporation 1996.

[34] Kankaanpaa A, Meririnne E, Ariniemi K, Seppala T. Oxalic acid stabilizes dopamine, serotonin, and their metabolites in automated 
liquid chromatography with electrochemical detection. J Chromatogr B Biomed Sci Appl 2001; 753: 413-9.

[35] Chen F, Larsen MB, Sanchez C, Wiborg O. The S-enantiomer of R, S-citalopram, increases inhibitor binding to the human serotonin transporter by an allosteric mechanism. Comparison with other serotonin transporter inhibitors. Eur Neuropsychopharmacol 2005; 15: 193-8.

[36] Widner B, Laich A, Sperner-Unterweger B, Ledochowski M, Fuchs D. Neopterin production, tryptophan degradation, and mental depression--what is the link? Brain Behav Immun 2002; 16: 590-5.

[37] Laich A, Neurauter G, Widner B, Fuchs D. More rapid method for simultaneous measurement of tryptophan and kynurenine by HPLC. Clin Chem 2002; 48: 579-81.

[38] Highleyman L, Franciscus A. HCV viral load tests. Available at: http://www.hcvadvocate.org/hepatitis/Basics/Viralload_09.pdf [Accessed August 3, 2008].

[39] Nocito A, Dahm F, Jochum W, et al. Serotonin mediates oxidative stress and mitochondrial toxicity in a murine model of nonalcoholic steatohepatitis. Gastroenterology 2007; 133: 608-18.

[40] Endo Y, Nakamura M. The effect of lipopolysaccharide, interleukin-1 and tumour necrosis factor on the hepatic accumulation of 5-hydroxytryptamine and platelets in the mouse. Br J Pharmacol 1992; 105: 613-9.

[41] Cressman DE, Greenbaum LE, DeAngelis RA, et al. Liver failure and defective hepatocyte regeneration in interleukin-6-deficient mice. Science 1996; 274: 1379-83.

[42] Durk T, Panther E, Muller T, et al. 5-Hydroxytryptamine modulates cytokine and chemokine production in LPS-primed human monocytes via stimulation of different 5-HTR subtypes. Int Immunol 2005; 17: 599-606.

[43] Nakamura N, Hamada N, Murata R, et al. Contribution of serotonin to liver injury following canine small-intestinal ischemia and reperfusion. J Surg Res 2001; 99: 17-24.

[44] Murata R, Hamada N, Nakamura N, et al. Serotonin activity and liver dysfunction following hepatic ischemia and reperfusion. In Vivo 2003; 17: 567-72.

[45] Lang PA, Contaldo C, Georgiev P, et al. Aggravation of viral hepatitis by platelet-derived serotonin. Nat Med 2008; 14: 756-61.
[46] Maneglier B, Guillemin GJ, Clayette P, et al. Serotonin decreases HIV-1 replication in primary cultures of human macrophages through 5-HT(1A) receptors. Br J Pharmacol 2008; 154: 174-82.

[47] Loftis JM, Morasco BJ, Menasco DJ, et al. Serotonin levels are associated with sustained viral response rates but not depressive symptoms in HCV patients undergoing interferon-based therapy: Presented: $13^{\text {th }}$ International Symposium on Viral Hepatitis and Liver Disease. Washington D.C. Mar 20-24; 2009.

[48] Larrea E, Riezu-Boj JI, Gil-Guerrero L, et al. Upregulation of indolamine 2,3-dioxygenase in hepatitis $\mathrm{C}$ virus infection. J Virol 2007; 81: 3662-6.

[49] Kraus MR, Al-Taie O, Schefer A, Pfersdorff M, Lesch KP, Scheurlen M. Serotonin-1A receptor gene (HTR1A) variation predicts interferon induced depression in chronic hepatitis $\mathrm{C}$. Gastroenterology 2007; 132: 1279-86.

[50] Lotrich FE, Ferrell RE, Rabinovitz M, Pollock BG. Risk for depression during interferon-alpha treatment is affected by the serotonin transporter polymorphism. Biol Psychiatry 2009; 65: 344-8.

[51] Robaeys G, Wichers MC, De Bie J, et al. Does antidepressant medication in patients with hepatitis $\mathrm{C}$ undergoing interferon alpha treatment reduce therapeutic efficacy? Gut 2009; 58:145

[52] Kraus MR, Schafer A, Scheurlen M. Therapy of interferon-induced depression in chronic hepatitis $\mathrm{C}$ with citalopram: a randomised, double-blind, placebo-controlled study. Gut 2008; 57(4): 531-6 [Comment in: Gut 2009; 58(1): 145; author reply 145-6].

[53] Lackner C, Struber G, Liegl B, et al. Comparison and validation of simple noninvasive tests for prediction of fibrosis in chronic hepatitis C. Hepatol 2005;41:1376-82.

[54] Wai C, Greenson JK, Fontana RJ, et al. A simple noninvasive index can predict both significant fibrosis and cirrhosis in patients with chronic hepatitis C. Hepatol 2003; 38:518-26.

[55] Shaheen AAM, Myers RP. Diagnostic accuracy of the aspartate aminotransferase-to-platelet ratio index for the prediction of hepatitis C-related fibrosis: a systematic review. Hepatology 2007; 46:912-21

(C) Loftis et al.; Licensee Bentham Open.

This is an open access article licensed under the terms of the Creative Commons Attribution Non-Commercial License (http://creativecommons.org/licenses/ by-nc/3.0/) which permits unrestricted, non-commercial use, distribution and reproduction in any medium, provided the work is properly cited. 